\title{
Numerical Study of Generalized Three-Dimensional MHD Flow over a Porous Stretching Sheet
}

\author{
M. Heydari ${ }^{1}$, G. B. Loghmani $^{1 \dagger}$ and A. A. Dehghan ${ }^{2}$ \\ ${ }^{I}$ Department of Mathematics, Yazd University, Yazd, Iran \\ ${ }^{2}$ School of Mechanical Engineering, Yazd University, Yazd, Iran \\ $†$ Corresponding Author Email: loghmani@yazd.ac.ir
}

(Received March 5, 2013; accepted June 9, 2013)

\begin{abstract}
In this paper a numerical method for solving generalized three-dimensional magnetohydrodynamic (MHD) flow of an incompressible viscous fluid over a porous stretching sheet is proposed. This approach is based on Legendre pseudo-spectral method with a positive scaling factor and extrapolation. The present method solves the problem on the semi-infinite domain without truncating it to a finite domain. In addition, this method reduces the solution of the problem to solution of a system of algebraic equations. The obtained numerical results are compared with some well-known results to confirm the accuracy and efficiency of the proposed scheme.
\end{abstract}

Keywords: Porous stretching sheet, Three-dimensional flow, Legendre polynomials, Pseudo-spectral method, Extrapolation, Scaling factor.

\section{NOMENCLATURE}

$\begin{array}{ll}a, b & \text { constants of proportionality } \\ B & \text { stretching ratio } \\ B_{0} & \text { magnetic field strength } \\ f, g & \text { dimensionless stream functions } \\ L & \text { map parameter } \\ L_{n}(\xi) & \text { Legendre polynomial of order } n \\ M^{2} & \text { Hartman number } \\ \mathrm{N} & \text { order of approximation } \\ R & \text { suction or injection parameter } \\ w_{0} & \text { suction or injection velocity } \\ p & \text { pressure }\end{array}$

\section{INTRODUCTION}

Many of the mathematical modeling of physical phenomena in science and engineering often lead to nonlinear differential equations or partial differential equations. Most of these problems are nonlinear, therefore, some of them are solved using numerical solution and some are solved using a different analytic method, such as Runge-Kutta method (Mahantiand Gaur (2009)), Euler method, Hybrid methods, meshless methods, spectral methods(Civalek (2006); Civalek (2007); Snyder (1966);Clenshaw and Curtis (1960)), the $\delta$ expansion method(Karmishin, Zhukov, and Kolosov (1990)) the Adomain decomposition method(Adomian (1994)), the variational iteration $\mathrm{p}_{0} \quad$ pressure at some reference point

$\mathrm{U}$ velocity component in the $\mathrm{x}$ direction

$\mathrm{v}$ velocity component in the $y$ direction

$\mathrm{w} \quad$ velocity component in the $\mathrm{z}$ direction

$\lambda$ eigenvalue

$\eta \quad$ similarity variable

$v \quad$ kinematic viscosity

$\rho \quad$ fluid density

$\sigma \quad$ electrical conductivity

method(He (1999); He (2000)), the homotopy perturbation method(He (2003); He (2004))and thehomotopy analysis method (Liao (2003); Xu, Liao, and Pop (2006)). Spectral methods are very powerful tools for solving many types of differential equations arising in various fields of science and engineering (Canuto, Hussaini, Quarteroni, and Zang (1988); Boyd (2001)). Exponential convergence and convenience of applying these methods are two effective properties which have encouraged many authors to use them for finding the approximate solution of different equations. The basic idea of spectral methods to solve differential equations is to expand the solution function as a finite series of very smooth basis functions, as given 
$u^{N}(\xi)=\sum_{n=0}^{N} u_{n} \phi_{n}(\xi)$

in which, the best choice of $\phi_{n}(\xi)$, are the eigenfunctions of a singular Sturm-Liouville problem.

The flow of an incompressible viscous fluid over a stretching sheet has an important bearing on several technological processes, like in the extrusion of a polymer in a melt spinning process and metallurgy. This problem first discovered by Crane (1970) for the two-dimensional flow. Since then the problem has been extensively studied by taking into account many different physical features either separately or in various combinations. Gupta and Gupta (1977) studied the heat and mass transfer in a stretching surface with suction or blowing. Chen and char (1988) studied the effects of variable surface temperature and variable surface heat flux on the heat transfer characteristics of a linearly stretching sheet. Afterwards, several researchers presented closed form solutions for the Crane's problem when suction, magnetic field, viscoelasticity of the fluid etc. are considered (Andersson (1992); Troy, II, Ermountrout, and Keener (1987); Ariel (1994)). Wang (Wang (1984)) considered the general threedimensional flow of a Newtonian fluid due to a stretching sheet. He also gave in (Wang (2002)) an exact solution for the two-dimensional flow due to a stretching boundary with a partial slip. Ariel (2003) obtained the perturbation and semi-analitical solution for generalized three-dimensional flow of a hydrodynamic fluid over a non-porous stretching sheet. Also a study on three-dimensional flow of a viscous fluid over a non-porous stretched surface using homotopy perturbation method is proposed by Ariel (2007). The magnetohydrodynamic (MHD) three-dimensional viscous flow over a porous stretching surface has been reported by Hayat and Javed (2007).

In this study, a combination of pseudo-spectral collocation method with a positive scaling factor and extrapolation is uesd to solve the generalized three-dimensional MHD flow over a porous stretching sheet subjected to a uniform suction and injection. The main point of the present analysis lies in the fact that the present method solves the problem on the semi-infinite domain without truncating it to a finite domain. The results are compared with those in the literature (Wang (1984); Ariel (2003); Ariel (2007); Hayat and Javed (2007)) that includes suction/injection and an applied magnetic field.

The paper is organized in the following pattern. In Section 2, the mathematical formulation is presented. Some necessary definitions and mathematical preliminaries of the Legendre polynomials are introduced in Section 3. In Section 4 , the proposed method is applied to solve generalized three-dimensional MHD flow over a porous stretching sheet. Results and comparisons with existing methods in the literature are presented in Section 5. In Section 6, some methods to choose the map parameter $L$ are introduced and finally conclusions are drawn in Section 7.

\section{PROBLEM STATEMENT AND MATHEMATICAL FORMULATION}

Consider the steady, laminar flow of an incompressible viscous fluid induced by the stretching of a porous surface in the plane $z=0$. Let $u, v$ and $w$ be the velocity components along $x, y$ and $z$ directions, respectively. The fluid is electrically conducting and it flows in the presence of an applied uniform magnetic field $\mathrm{B}_{0}$. The fluid injection or suction takes place through the porous surface with velocity $-w_{0}$ where $w_{0}>0$ corresponds to the suction velocity and $w_{0}<0$ holds for injection velocity. The velocity $(u, v, w)$ at any point of the porous stretching sheet is given by

$u=a x, v=b y, w=-w_{0}$ at $z=0$,

wherea and $\mathrm{b}$ are the constants of proportionality with dimensional of $s^{-1}$. By using the similarity transforms (Hayat and Javed2007)

$u=\operatorname{axf} f^{\prime}(\eta), \quad v=a y g^{\prime}(\eta)$,

$w=-\sqrt{a v}(f(\eta)+g(\eta))$,

where $v$ is the coefficient of kinematic viscosity and

$\eta=\sqrt{\frac{a}{v}} Z$

is the similarty variable, the Navier-Stokes equations reduce to the following set of ODE's:

$f^{\prime \prime \prime}+(f+g) f^{\prime \prime}-\left(f^{\prime}\right)^{2}-M^{2} f^{\prime}=0$,

$g^{\prime \prime \prime}+(f+g) g^{\prime \prime}-\left(g^{\prime}\right)^{2}-M^{2} g^{\prime}=0$.

Here, $\mathrm{M}^{2}=\frac{\sigma \mathrm{B}_{0}^{2}}{\rho \mathrm{a}}$ is the Hartman number, $\rho$ is the

fluid density and $\sigma$ is theelectrical conductivity. The boundary conditions for the problem are:

$f(0)+g(0)=R, f^{\prime}(0)=1, g^{\prime}(0)=b / a=B$,

$f^{\prime}(\infty)=0, \quad g^{\prime}(\infty)=0$,

where

$R=\frac{w_{0}}{\sqrt{a v}}$

and prime denotes the derivative with respect to $\eta$. The pressure $\mathrm{p}$ can be recovered by

$\mathrm{p}=\mathrm{p}_{0}+\rho\left(v \mathrm{w}_{\mathrm{z}}-\frac{1}{2} \mathrm{w}^{2}\right)$,

where $p_{0}$ is the pressure at some reference point (the origin in the present problem). 
M. Heydari et al. / JAFM, Vol. 7, No. 3, pp. 473-483, 2014.

\section{SOME PRELIMINARIES}

\subsection{Properties of Legendre polynomials}

Orthogonal polynomials are widely used in many areas such as mathematical physics, engineering and computer science (El-Mikkawy and Cheon (2005); Maleknejad and Kajani (2003)). One of the most common polynomials is a set of Legendre polynomials $\left\{L_{n}(\xi)\right\}_{n=0}^{\infty}$ which are orthogonal on the interval $[-1,1]$ with respect to the weight function $w(\xi)=1$, (Datta and B.M.Mohan 1995). In mathematics, Legendre polynomials are eigenfunctions of singular Sturm-Liouville problem

$\frac{d}{d \xi}\left[\left(1-\xi^{2}\right) \frac{d}{d \xi} L_{n}(\xi)\right]+\lambda L_{n}(\xi)=0$,

where the eigenvalue $\lambda$ equals $n(n+1)$. These polynomials satisfy therecurrence relation (Datta andB.M.Mohan (1995))

$$
\begin{aligned}
& (n+1) L_{n+1}(\xi)=(2 n+1) \xi L_{n}(\xi)-n L_{n-1}(\xi) \\
& n=1,2, \cdots
\end{aligned}
$$

with

$$
L_{0}(\xi)=1, \quad L_{1}(\xi)=\xi
$$

The orthogonality of these polynomials is expressed by the relation

$$
\int_{-1}^{1} L_{n}(\xi) L_{m}(\xi) d \xi=\frac{2}{2 n+1} \delta_{m, n} .
$$

Square integrable function $u(\xi) \operatorname{in}[-1,1]$, may be expressed in terms ofLegendre polynomials as

$u(\xi)=\sum_{j=0}^{\infty} u_{j} L_{j}(\xi)$,

where the coefficients $u_{j}$ are given by

$u_{j}=\frac{2 j+1}{2} \int_{-1}^{1} L_{j}(\xi) u(\xi) d \xi, \quad j=0,1,2, \cdots$

\subsection{Theorem}

Canuto, Hussaini, Quarteroni, and Zang (1988) Let $u(\xi) \in H^{k}(-1,1)$ (Sobolev space), $u^{N}(\xi)=$ $\sum_{j=0}^{N} u_{j} L_{j}(\xi)$ be the best approximation polynomial of $\mathrm{u}(\xi)$ in $L_{2}$-norm. Then

$$
\left\|u(\xi)-u^{N}(\xi)\right\|_{L_{2}[-1,1]} \leq \frac{C_{0}}{N^{k}}\|u(\xi)\|_{H^{k}(-1,1)},
$$

where $C_{0}$ is a positive constant, which depends on the selected norm and is independent of $u(\xi)$ and $N$.Let $\left\{\xi_{i}\right\}_{i=0}^{N}$ denote the Legendre-Gauss-Lobatto nodes (LGL nodes). These grids $\xi_{0}=-1<\xi_{1}<$ $\cdots<\xi_{N-1}<\xi_{N}=1$ are the zeros of $(1-$ $\left.\xi^{2}\right) L_{N}^{\prime}(\xi)$. Canuto et al. (1988) introduced the following approximation of the functionu( $(\xi)$,

$u^{N}(\xi)=\sum_{j=0}^{N} \tilde{u}_{j} L_{j}(\xi)$,

where $\tilde{u}_{j}$ are the Legendre coefficients which are determined by the following formulations $\tilde{u}_{j}=\frac{1}{\gamma_{j}} \sum_{i=0}^{N} u\left(\xi_{i}\right) L_{j}\left(\xi_{i}\right) w_{i}, \quad j=0,1, \cdots, N$,

with

$w_{i}=\frac{2}{N(N+1)\left[L_{N}\left(\xi_{i}\right)\right]^{2}}, \quad i=0,1, \cdots, N$,

and

$\gamma_{j}=\left\{\begin{array}{lr}\frac{2}{2 j+1}, & 0 \leq j \leq N-1, \\ \frac{2}{N}, & j=N .\end{array}\right.$

As it is well-known in Legendre pseudo-spectral method, derivatives of thefunctions $u(\xi)$ at the collocation points are presented as

$\frac{\mathrm{du}}{\mathrm{d} \xi}\left(\xi_{\mathrm{i}}\right)=\sum_{\mathrm{j}=0}^{\mathrm{N}} \mathcal{D}_{\mathrm{ij}} \mathrm{u}\left(\xi_{\mathrm{j}}\right)$,

$\frac{d^{2} u}{d \xi^{2}}\left(\xi_{i}\right)=\sum_{j=0}^{N} \mathcal{D}_{i j}^{(2)} u\left(\xi_{j}\right)$.

In the above equations $\mathcal{D}$ is the Legendre differentiation matrix and $N+1$ is the number of collocation points (nodes) and $\mathcal{D}^{(2)}=(\mathcal{D})^{2}$. The entries of the differentiation matrix $\mathcal{D}$ are given by Canuto et al. (1988)

$\mathcal{D}_{i j}=\left\{\begin{array}{cc}-\frac{N(N+1)}{4}, & i=j=0, \\ \frac{N(N+1)}{4}, & i=j=N, \\ 0, & 1 \leq i=j \leq N-1, \\ \frac{1}{\xi_{i}-\xi_{j}} \frac{L_{N}\left(\xi_{i}\right)}{L_{N}\left(\xi_{j}\right)}, & i \neq j, 0 \leq i, j \leq N .\end{array}\right.$

\subsection{Mappings}

A common and effective method for solving a differential equation with unbounded domain is to use a suitable mapping that transforms a problem with infinite domain to a problem with finite domain (Boyd (2001); Shen and Wang (2009)).

We consider a family of mappings as follows:

$\eta=g(\xi ; L)$,

$\xi \in I=(-1,1), \quad \eta \in \Lambda=(0,+\infty)$,

such that

$\frac{d \eta}{d \xi}=g^{\prime}(\xi ; L)>0, \quad \xi \in I=(-1,1)$
$g(-1 ; L)=0, \quad g(1 ; L)=+\infty$.

Here, the parameter $L$ is a positive scaling factor that is called mapping parameter. Without loss of generality, we further assume that the mapping is explicitly invertible, and denote its inverse mapping by $\xi=g^{-1}(\eta ; L)$

$\eta \in \Lambda=(0,+\infty), \quad \xi \in I=(-1,1)$.

There are several typical mappings that relate infinite and finite domains to each other, but algebraic, logarithmic, and exponential mapping 
functions are more practical. Typical examples of such functions are:

- The algebraic mapping

$\xi=\frac{\eta-L}{\eta+L}, \quad \eta=L \frac{1+\xi}{1-\xi}$.

- The logarithmic mapping

$\xi=2 \tanh \left(\frac{\eta}{L}\right)-1, \quad \eta=\frac{L}{2} \ln \left(\frac{3+\xi}{1-\xi}\right)$.

- The exponential mapping

$\xi=\frac{2}{L} \ln \left(\eta+\sqrt{\eta^{2}+1}\right)-1$,

$\eta=\sinh \left(\frac{L}{2}(1+\xi)\right)$

where $\xi \in I=(-1,1)$ and $\eta \in(0, \tilde{L})$ with $\tilde{L}=$ $\sinh (L)$.

Boyd (2001, 1982) offered guidelines for optimizing the map parameter $L$ for rational Chebyshev functions, which is useful for the presented method in this paper, too. For the map parameter $L$ we have the following results (Boyd 2001, 1982):

- In general, there is no way to avoid a small amount of trial and error in choosing $L$ when solving problems on an infinite domain

- $\quad$ The optimum value of $L$ varies with the number of collocation points

- A little experimentation is usually sufficient to determine a suitable value of $L$ because near the optimum value of $L$, the accuracy is insensitive to the precise value of $L$

- The parameter $L$ is a scaling/stretching factor which can be used to fine tune the spacing of collocation points

The mappings in Eqs. (28) to (30) are especially convenient because they yield simple expressions for derivatives. For a general mapping $\eta=g(\xi ; L)$ with $g^{\prime}(\xi ; L)>0, \quad \xi \in I=(-1,1)$, the first and second derivatives of $U(\eta)$ can be expressed in terms of $\xi$ as follows:

$\frac{d U}{d \eta}=\left(\frac{d g(\xi ; L)}{d \xi}\right)^{-1} \frac{\mathrm{dU}}{\mathrm{d} \xi}$

$\frac{d^{2} U}{d \eta^{2}}=\left(\frac{d g(\xi ; L)}{d \xi}\right)^{-2} \frac{d^{2} U}{d \xi^{2}}-\left(\frac{d^{2} g(\xi ; L)}{d \xi^{2}}\right)\left(\frac{d g(\xi ; L)}{d \xi}\right)^{-3} \frac{d U}{d \xi}$.

In a special case for the logarithmic mapping (29), the Eqs. (31) and (32) can be writen as follows:

$\frac{d U}{d \eta}=\frac{2}{L}\left[1-\left(\frac{1+\xi}{2}\right)^{2}\right] \frac{d U}{d \xi}$,

$\frac{d^{2} U}{d \eta^{2}}=\frac{4}{L^{2}}\left[1-\left(\frac{1+\xi}{2}\right)^{2}\right]^{2} \frac{d^{2} U}{d \xi^{2}}$

$-\frac{4}{L^{2}}\left(\frac{\xi+1}{2}\right)\left[1-\left(\frac{1+\xi}{2}\right)^{2}\right] \frac{d U}{d \xi}$.

\section{THE METHOD OF SOLUTION}

The present work deals with application of Legendre pseudo-spectral collocation method and extrapolation for solving generalized threedimensional magnetohydrodynamic (MHD) flow of an incompressible viscous fluid over a porous stretching sheet. This method solves the problem on the semi-infinite domain without truncating it to a finite domain. If semi-infinite domain $[0,+\infty)$ truncation to a domain $\left[0, \eta_{\infty}\right]$ is employed, then one must choose $\eta_{\infty}$. If the rate of decay of $U(\eta)$ for large values of $\eta$ is known or can be expected apriori, then $\eta_{\infty}$ may be choosen so that $U\left(\eta_{\infty}\right)<$ $\varepsilon$ where $\varepsilon$ is some user-chosen tolerance. But then one is still faced with choosing the grid spacing $h$ so that the error in solving the differential equation on interval $\left[0, \eta_{\infty}\right]$ is small. If $h$ is small, the error in solving the problem may be very much less than $\varepsilon$, in that case one has made a bad choice of $\eta_{\infty}$ because the domain truncation error is dominant, and it would have been better to choose a larger $\eta_{\infty}$. In this paper, the logarithmic mapping (29) for converting semi-infinite domain $[0,+\infty)$ into the computational domain $(-1,1)$ is used.

We define

$f^{\prime}=\frac{F+G}{2}, \quad g^{\prime}=\frac{F-G}{2}, \quad f+g=H$.

Ariel (2003) applied the above transforms for solving generalized three-dimensional flow of a hydrodynamic fluid over a non-porous stretching sheet by using Ackroyd's series method (Ackroyd 1978). Here, we apply Eq. (35) for solving generalized three-dimensional magnetohydrodynamic fluid over a porous stretching sheet. By using Eq. (35), Eqs. (4) to (7) transform to :

$H^{\prime}-F=0$,

$F^{\prime \prime}+H F^{\prime}-\frac{1}{2}\left(F^{2}+G^{2}\right)-M^{2} F=0$,

$G^{\prime \prime}+H G^{\prime}-F G-M^{2} G=0$,

subjected to the boundary conditions

$F(0)=1+B, \quad G(0)=1-B, \quad H(0)=R$,

$F(\infty)=0, \quad G(\infty)=0$.

By using Eqs. (33) and (34), Eqs. (36) to (40) are converted to the differential equations with boundary conditions on interval $[-1,1]$ as follows:

$\frac{2}{L}\left[1-\left(\frac{1+\xi}{2}\right)^{2}\right] \frac{d H}{d \xi}-F=0$,

$\frac{4}{L^{2}}\left[1-\left(\frac{1+\xi}{2}\right)^{2}\right]^{2} \frac{d^{2} F}{d \xi^{2}}-\frac{4}{L^{2}}\left(\frac{\xi+1}{2}\right)\left[1-\left(\frac{1+\xi}{2}\right)^{2}\right] \frac{d F}{d \xi}$

$+\frac{2}{L}\left[1-\left(\frac{1+\xi}{2}\right)^{2}\right] \frac{d F}{d \xi} H-\frac{1}{2}\left(F^{2}+G^{2}\right)-M^{2} F=$

0 ,

$\frac{4}{L^{2}}\left[1-\left(\frac{1+\xi}{2}\right)^{2}\right]^{2} \frac{d^{2} G}{d \xi^{2}}-\frac{4}{L^{2}}\left(\frac{\xi+1}{2}\right)\left[1-\left(\frac{1+\xi}{2}\right)^{2}\right] \frac{d G}{d \xi}+$

$\frac{2}{L}\left[1-\left(\frac{1+\xi}{2}\right)^{2}\right] \frac{d G}{d \xi} H-F G-M^{2} G=0$,

and

$F(-1)=1+B, G(-1)=1-B, H(-1)=R,(44)$

$F(1)=0, \quad G(1)=0$.

Now, Legendre pseudo-spectral method is utilized to solve the above problem as follows. This method involves using the Legendre-Gauss-Lobatto points to discrete interval $[-1,1]$. Let the unknown functions $F(\xi), G(\xi)$ and $H(\xi)$ be approximated by a truncated series of Legendre polynomials as

$F(\xi) \simeq F^{N}(\xi)=\sum_{j=0}^{N} \tilde{f}_{j} L_{j}(\xi)$, 
M. Heydari et al. / JAFM, Vol. 7, No. 3, pp. 473-483, 2014.

$G(\xi) \simeq G^{N}(\xi)=\sum_{j=0}^{N} \tilde{g}_{j} L_{j}(\xi)$,
$H(\xi) \simeq H^{N}(\xi)=\sum_{j=0}^{N} \tilde{h}_{j} L_{j}(\xi)$,

where $\tilde{f}_{j}, \tilde{g}_{j}$ and $\tilde{h}_{j}$ are the Legendre coefficients which are determined by the formulations

$\tilde{f}_{j}=\frac{1}{\gamma_{j}} \sum_{i=0}^{N} F\left(\xi_{i}\right) L_{j}\left(\xi_{i}\right) w_{i}, \quad j=0,1, \cdots, N$,

$\tilde{g}_{j}=\frac{1}{\gamma_{j}} \sum_{i=0}^{N} G\left(\xi_{i}\right) L_{j}\left(\xi_{i}\right) w_{i}, \quad j=0,1, \cdots, N$,

$\tilde{h}_{j}=\frac{1}{\gamma_{j}} \sum_{i=0}^{N} H\left(\xi_{i}\right) L_{j}\left(\xi_{i}\right) w_{i}, \quad j=0,1, \cdots, N$.

By employing derivatives formulations (21) and (22), Eqs. (41) to (45) are transformed to the following expressions

$$
\begin{aligned}
& \frac{2}{L}\left[1-\left(\frac{1+\xi_{i}}{2}\right)^{2}\right] \sum_{j=0}^{N} \mathcal{D}_{i j} H\left(\xi_{j}\right)-F\left(\xi_{i}\right)=0, \\
& \frac{4}{L^{2}}\left[1-\left(\frac{1+\xi_{i}}{2}\right)^{2}\right]^{2} \sum_{j=0}^{N} \mathcal{D}_{i j}^{2} F\left(\xi_{j}\right) \\
& -\frac{4}{L^{2}}\left(\frac{\xi_{i}+1}{2}\right)\left[1-\left(\frac{1+\xi_{i}}{2}\right)^{2}\right] \sum_{j=0}^{N} \mathcal{D}_{i j} F\left(\xi_{j}\right) \\
& +\frac{2}{L}\left[1-\left(\frac{1+\xi_{i}}{2}\right)^{2}\right] \sum_{j=0}^{N} \mathcal{D}_{i j} F\left(\xi_{j}\right) H\left(\xi_{i}\right) \\
& -\frac{1}{2}\left(F^{2}\left(\xi_{i}\right)+G^{2}\left(\xi_{i}\right)\right)-M^{2} F\left(\xi_{i}\right)=0, \\
& \frac{4}{L^{2}}\left[1-\left(\frac{1+\xi_{i}}{2}\right)^{2}\right]^{2} \sum_{j=0}^{N} \mathcal{D}_{i j}^{2} G\left(\xi_{j}\right) \\
& -\frac{4}{L^{2}}\left(\frac{\xi_{i}+1}{2}\right)\left[1-\left(\frac{1+\xi_{i}}{2}\right)^{2}\right] \sum_{j=0}^{N} \mathcal{D}_{i j} G\left(\xi_{j}\right)
\end{aligned}
$$$$
+\frac{2}{L}\left[1-\left(\frac{1+\xi_{i}}{2}\right)^{2}\right] \sum_{j=0}^{N} \mathcal{D}_{i j} G\left(\xi_{j}\right) H\left(\xi_{i}\right)
$$$$
-F\left(\xi_{i}\right) G\left(\xi_{i}\right)-M^{2} G\left(\xi_{i}\right)=0 \text {, }
$$

where $i=1,2, \cdots, N-1$ and

$$
F\left(\xi_{0}\right)=1+B \quad, G\left(\xi_{0}\right)=1-B, \quad H\left(\xi_{0}\right)=R,(55)
$$$$
F\left(\xi_{N}\right)=0, \quad G\left(\xi_{N}\right)=0 \text {. }
$$

For finding approximate solutions $F(\xi), G(\xi)$ and $H(\xi)$, we need to calculate values $\left\{F\left(\xi_{i}\right)\right\}_{i=1}^{N-1},\left\{G\left(\xi_{i}\right)\right\}_{i=1}^{N-1}$ and $\left\{H\left(\xi_{i}\right)\right\}_{i=1}^{N}$. However, the Eqs. (52) to (54) give a system with $3 N-3$ equations and $3 N-2$ unknowns. To construct the remaining one equation we can use extrapolation method. Extrapolating is defined as estimating a point outside a known data set. Here, we consider $\mathrm{N}$ datapoints

$$
\left\{\left(\xi_{0}, H\left(\xi_{0}\right)\right),\left(\xi_{1}, H\left(\xi_{1}\right)\right), \cdots,\left(\xi_{N-1}, H\left(\xi_{N}-1\right)\right)\right\},
$$

generated by the unknown function $H(\xi)$. So, we can interpolate $H(\xi)$ by the Lagrange form of the interpolation polynomial as follows:

$H(\xi) \simeq \sum_{j=0}^{N-1} H\left(\xi_{j}\right) L_{j}(\xi)$,

where

$$
L_{j}(\xi)=\prod_{k=0, k \neq j}^{N-1} \frac{\xi-\xi_{k}}{\xi_{j}-\xi_{k}}, \quad j=0,1, \cdots, N-1,
$$

are Lagrange polynomials. By collocating Eq. (57) in point $\xi_{N}$, we obtain

$H\left(\xi_{N}\right)-\sum_{j=0}^{N-1} H\left(\xi_{j}\right) L_{j}\left(\xi_{N}\right)=0$.

Equations (52) to (54) together with Eq. (59) give a $(3 N-2) \times(3 N-2) \quad$ system of nonlinear equations, which can be solved for $\left\{F\left(\xi_{i}\right)\right\}_{i=1}^{N-1},\left\{G\left(\xi_{i}\right)\right\}_{i=1}^{N-1}$ and $\left\{H\left(\xi_{i}\right)\right\}_{i=1}^{N}$, using Newton's iterative method (Ortega and Rheinboldt 1970; Kelley 1995). After evaluating $F(\xi), G(\xi)$ and $H(\xi)$, the original functions $F(\eta), G(\eta)$ and $H(\eta)$ are obtained by using Eqs. (46) to (48) and (29) as follows:

$$
\begin{aligned}
& F(\eta) \simeq F^{N}(\eta)=\sum_{j=0}^{N} \tilde{f}_{j} L_{j}\left(2 \tanh \left(\frac{\eta}{L}\right)-1\right),(60) \\
& G(\eta) \simeq G^{N}(\eta)=\sum_{j=0}^{N} \tilde{g}_{j} L_{j}\left(2 \tanh \left(\frac{\eta}{L}\right)-1\right),(61) \\
& H(\eta) \simeq H^{N}(\eta)=\sum_{j=0}^{N} \tilde{h}_{j} L_{j}\left(2 \tanh \left(\frac{\eta}{L}\right)-1\right) .(62)
\end{aligned}
$$

\subsection{Remark}

The values of $f^{\prime \prime}(0)$ and $g^{\prime \prime}(0)$ can be obtained from Eqs. (60) to (62) and (35) as follows:

$$
\begin{aligned}
& f^{\prime \prime}(0) \simeq \frac{1}{2 L} \sum_{j=0}^{N}(-1)^{j-1} j(j+1)\left(\tilde{f}_{j}+\tilde{g}_{j}\right), \\
& g^{\prime \prime}(0) \simeq \frac{1}{2 L} \sum_{j=0}^{N}(-1)^{j-1} j(j+1)\left(\tilde{f}_{j}-\tilde{g}_{j}\right) .
\end{aligned}
$$

We note that $L_{n}^{\prime}( \pm 1)=( \pm 1)^{n-1} \frac{n(n+1)}{2}$.

\section{RESULTS AND DISCUSSION}

In this section the numerical and graphical results obtained by employing pseudo-spectral collocation method are presented for the problem under consideration. This section describes the influence of some interesting parameters on the velocity components $f^{\prime}(\eta)$ and $g^{\prime}(\eta)$. In particular, attention has been focused to the variations of the MHD non-dimensional parameter $M$, uniform injection (suction) parameter $R$ and stretching ratio parameter $B$. The current results are compared with the previously published results by (Ariel 2003; Ariel 2007; Wang 1984 ; Hayat and Javed 2007).

Tables 1 and 2 provide a comparative results of presented method $(N=22$ and $L=5)$ with exact solution (given by Ariel (2003)), Wang method (Wang 1984), HPM (Ariel 2007) and HAM (Hayat and Javed 2007) versus different values of stretching ratio $B$. It is found that the obtained solution from pseudo-spectral collocation method has a good agreement with the presented exact solutions in (Ariel 2003).

Table 3 displays the numerical results for $-f^{\prime \prime}(0)$ and $-g^{\prime \prime}(0)$ for different values of $M$ and $R$. This table shows that the magnitude of shear stresses increases for large value of $M$. Moreover, Table 3 indicates that the behavior of $R$ and $M$ on the magnitude of shear stresses is the same.

Figures. 1 to 6 have been made in order to see the effects of the MHD parameter $M$ and 
suction/injection parameter $R$ on the on the velocity profiles $f^{\prime}(\eta)$ and $g^{\prime}(\eta)$.

Figures. 1 and 2 are made to see the effect of the suction and injection velocity $R$ on the velocity profiles $f^{\prime}(\eta)$ and $g^{\prime}(\eta)$, respectively. It is found from them that the value of $f^{\prime}(\eta)$ and $g^{\prime}(\eta)$ decreases by increasing the value of $\mathrm{R}$ for the suction case $(R>0)$. However by increasing the value of $\mathrm{R}$ for the injection case $(R<0)$, the value of $f^{\prime}(\eta)$ and $g^{\prime}(\eta)$ increases.

Figures. 3 to 6 are sketched in order to see the effects of MHD parameter $M$ on the velocity profiles $f^{\prime}(\eta)$ and $g^{\prime}(\eta)$. These figures show that $f^{\prime}(\eta)$ and $g^{\prime}(\eta)$ decreases by increasing $M$ for both case of suction and injection. From Figs. 1 to 6 , it is seen a good agreement between the results obtained by Hayat et al. (2007)and presented method.

Table 1 Illustrating the variation of shear stress $-f^{\prime \prime}(0)$ with $B$ when $M=R=0, L=5, N=22$, using exact solution (Ariel 2007), the first order of perturbation solution in B (Wang 1984), HPM (Ariel 2007), HAM with $h=-0.75$ (Hayat and Javed 2007) and present method.

\begin{tabular}{|c|c|c|c|c|c|}
\hline B & Exact(Ariel 2007) & Wang(1988) & HPM (Ariel 2007) & HAM (Hayat and Javed 2007) & Present method \\
\hline 0 & 1 & 1 & 1 & 1 & 1 \\
\hline 0.1 & 1.02025978 & 1.020902 & 1.017027 & 1.02025978 & 1.0202597787 \\
\hline 0.2 & 1.03949519 & 1.041804 & 1.034587 & 1.03949519 & 1.0394951874 \\
\hline 0.3 & 1.05795478 & 1.062705 & 1.052470 & 1.05795478 & 1.0579547788 \\
\hline 0.4 & 1.07578811 & 1.083607 & 1.070529 & 1.07578811 & 1.0757881081 \\
\hline 0.5 & 1.09309502 & 1.104509 & 1.088662 & 1.09309502 & 1.0930950216 \\
\hline 0.6 & 1.10994694 & 1.125411 & 1.106797 & 1.10994694 & 1.1099469380 \\
\hline 0.7 & 1.12639752 & 1.146312 & 1.124882 & 1.12639752 & 1.1263975227 \\
\hline 0.8 & 1.14248863 & 1.167214 & 1.142879 & 1.14248863 & 1.1424886241 \\
\hline 0.9 & 1.15825383 & 1.188116 & 1.160762 & 1.15825383 & 1.1582538315 \\
\hline 1.0 & 1.17372074 & 1.209018 & 1.178511 & 1.17372074 & 1.1737207371 \\
\hline
\end{tabular}

Table 2 Illustrating the variation of shear stress $-g^{\prime \prime}(0)$ with $B$ when $M=R=0, L=5, N=22$, using exact solution (Ariel 2007), the first order of perturbation solution in B (Wang 1984), HPM (Ariel 2007) , HAM with $h=-0.75$ (Hayat and Javed 2007) and present method.

\begin{tabular}{|c|c|c|c|c|c|}
\hline B & Exact(Ariel 2007) & Wang(1988) & HPM (Ariel 2007) & HAM (Hayat and Javed 2007) & Present method \\
\hline 0 & 0 & 0 & 0 & 0 & 0 \\
\hline 0.1 & 0.06684715 & 0.058198 & 0.073099 & 0.06684715 & 0.0668471500 \\
\hline 0.2 & 0.14873691 & 0.116395 & 0.158231 & 0.14873691 & 0.1487369103 \\
\hline 0.3 & 0.24335980 & 0.174593 & 0.254347 & 0.24335980 & 0.2433598003 \\
\hline 0.4 & 0.34920865 & 0.232791 & 0.360599 & 0.34920865 & 0.3492086539 \\
\hline 0.5 & 0.46520485 & 0.290988 & 0.476290 & 0.46520485 & 0.4652048450 \\
\hline 0.6 & 0.59052892 & 0.349186 & 0.600833 & 0.59052892 & 0.5905289234 \\
\hline 0.7 & 0.72453174 & 0.407384 & 0.733730 & 0.72453174 & 0.7245317423 \\
\hline 0.8 & 0.86668292 & 0.465581 & 0.874551 & 0.86668292 & 0.8666829206 \\
\hline 0.9 & 1.01653870 & 0.523779 & 1.022922 & 1.01653870 & 1.0165386976 \\
\hline 1.0 & 1.17372074 & 0.581977 & 1.178511 & 1.17372074 & 1.1737207371 \\
\hline
\end{tabular}


M. Heydari et al. / JAFM, Vol. 7, No. 3, pp. 473-483, 2014.

Table 3 Illustrating the variation of shear stresses $-f^{\prime \prime}(0)$ and $-g^{\prime \prime}(0)$ with $R$ and $M$ using HAM with $h=-0.75$ (Hayat and Javed 2007) and present method when $B=0.1, L=5, N=22$.

\begin{tabular}{|c|c|c|c|c|c|}
\hline \multirow{2}{*}{$\mathrm{R}$} & \multirow{2}{*}{$\mathrm{M}$} & \multicolumn{2}{|c|}{$-f^{\prime \prime}(0)$} & \multicolumn{2}{|c|}{$-g^{\prime \prime}(0)$} \\
\hline & & $\begin{array}{l}\text { HAM (Hayat and Javed } \\
\text { 2007) }\end{array}$ & Present method & $\begin{array}{l}\text { HAM (Hayat and Javed } \\
\text { 2007) }\end{array}$ & Present method \\
\hline 0.1 & 0.1 & 1.076706 & 1.0767060574 & 0.736930 & 0.0736928293 \\
\hline 0.2 & 0.1 & 1.130707 & 1.1307074508 & 0.080087 & 0.0800875795 \\
\hline 0.3 & 0.1 & 1.187076 & 1.1870766613 & 0.086739 & 0.0867399461 \\
\hline 0.4 & 0.1 & 1.245772 & 1.2457719542 & 0.093634 & 0.0936343761 \\
\hline 0.5 & 0.1 & 1.306738 & 1.3067376319 & 0.100755 & 0.1007552295 \\
\hline 0.6 & 0.1 & 1.369906 & 1.3699056928 & 0.108087 & 0.1080870403 \\
\hline 0.7 & 0.1 & 1.435198 & 1.4351977919 & 0.115615 & 0.1156147281 \\
\hline 0.8 & 0.1 & 1.502527 & 1.5025273405 & 0.123324 & 0.1233237604 \\
\hline 0.9 & 0.1 & 1.571802 & 1.5718016324 & 0.131200 & 0.1312002722 \\
\hline 1.0 & 0.1 & 1.642924 & 1.6429238915 & 0.139231 & 0.1392311476 \\
\hline 0.1 & 0.2 & 1.091077 & 1.0910765549 & 0.075774 & 0.0757743773 \\
\hline 0.1 & 0.3 & 1.114617 & 1.1146167097 & 0.079125 & 0.0791258049 \\
\hline 0.1 & 0.4 & 1.146759 & 1.1467592420 & 0.083597 & 0.0835972841 \\
\hline 0.1 & 0.5 & 1.186797 & 1.1867966464 & 0.089021 & 0.0890208190 \\
\hline 0.1 & 0.6 & 1.233945 & 1.2339454036 & 0.095232 & 0.0952321624 \\
\hline 0.1 & 0.7 & 1.287404 & 1.2874043448 & 0.102084 & 0.1020842920 \\
\hline 0.1 & 0.8 & 1.346400 & 1.3463995504 & 0.109453 & 0.1094529523 \\
\hline 0.1 & 0.9 & 1.410214 & 1.4102137483 & 0.117237 & 0.1172368672 \\
\hline 0.1 & 1.0 & 1.478202 & 1.4782017749 & 0.125355 & 0.1253552221 \\
\hline
\end{tabular}

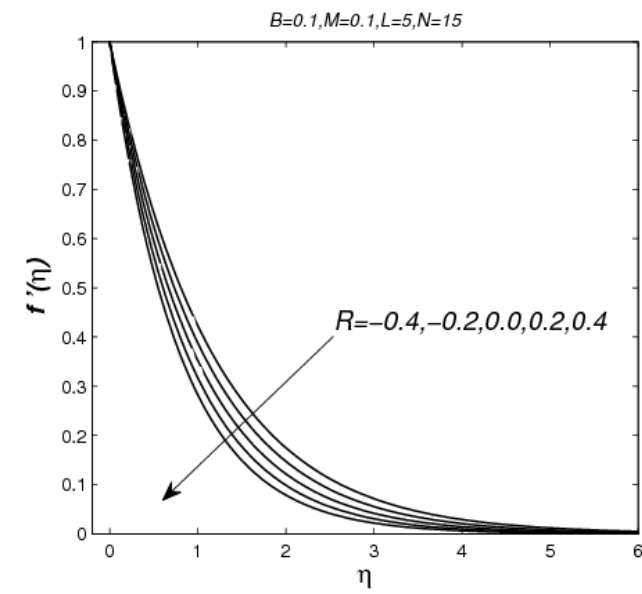

Fig. 1. Variation of similarity function $f^{\prime}$ with the increase in suction or injection parameterR.

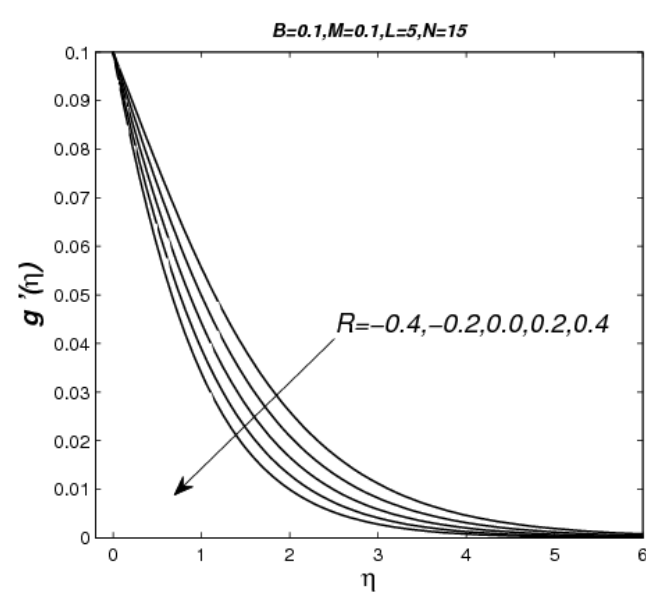

Fig. 2. Variation of similarity function $\mathrm{g}^{\prime}$ with the increase in suction or injection parameter R. 


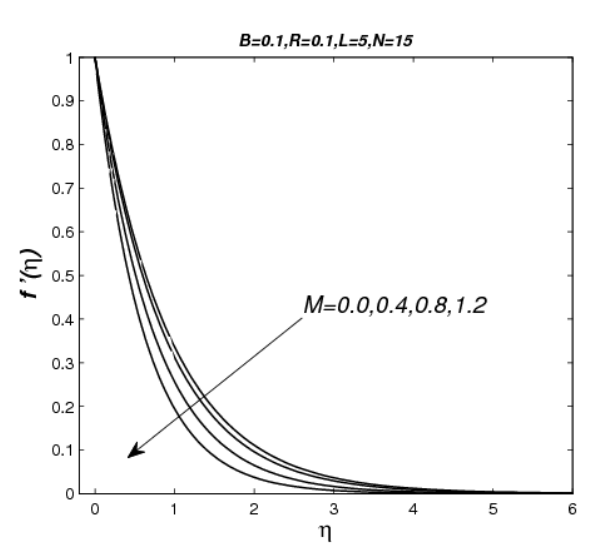

Fig. 3. Variation of similarity function $f^{\prime}$ with the increase in magnetic field parameter $M$ whenR $>0$.

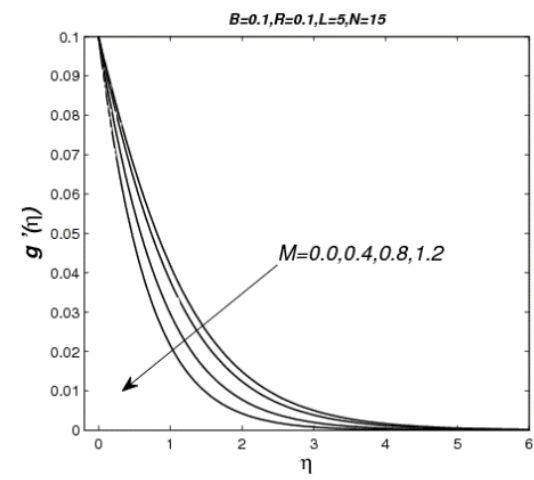

Fig. 4. Variation of similarity function $g^{\prime}$ with the increase in magnetic field parameter $M$ when $R>0$.

\section{THE CHOICE OF THE MAP PARAMETER $L$}

In general, there is not a simple method to choose the map parameter $\mathrm{L}$ for different problems, but we need to choose the map parameter $L$ such that it adjusts the width of the basis functions to the width of the solution. There are some methods for making this choice as outlined in (Boyd 2001, 1982,1994,1987), but the trial-and-error method is inevitable. Here, we introduce two strategies for finding a suitable map parameter L.

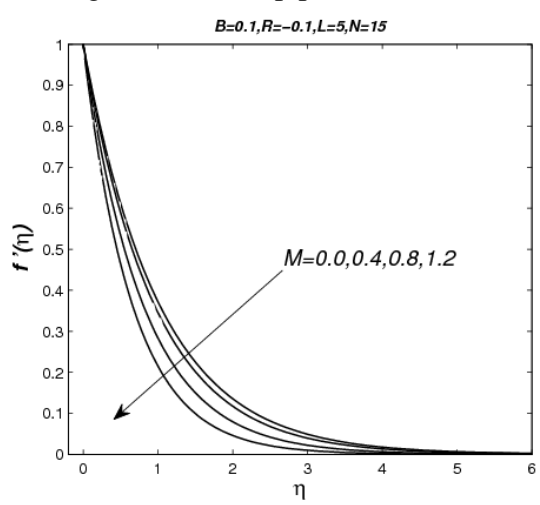

Fig. 5. Variation of similarity function $f^{\prime}$ with the increase in magnetic field parameter $M$ when $\boldsymbol{R}<\mathbf{0}$.

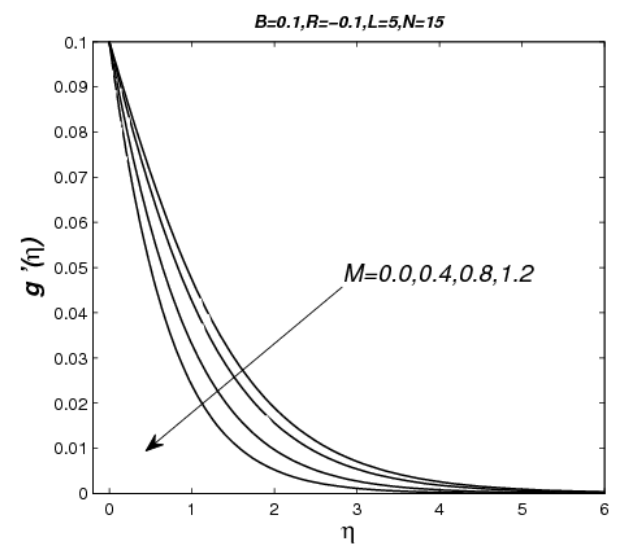

Fig. 6. Variation of similarity function $g^{\prime}$ with the increase in magnetic field parameter $M$ when $\boldsymbol{R}<\mathbf{0}$.

- The first strategy is to simply solve the problem and look at the spectral coefficients, plotted as absolute values on a log-linear plot, for several different values of $L$. Obviously, if the magnitudes of the coefficients are rapidly decreasing, the spectral series is accurate (Boyd 2001, 1982).

- The second strategy is to plot the residual error versus $\mathrm{L}$ for a fixed $\mathrm{N}$. This curve has a "V-shape" for reasons explained in more detail in (Boyd 2001,1982, 1987).

For the system of differential Eqs (4) and (5), the residual errors are defined as follows:

$$
\begin{aligned}
& R E S_{f}=f^{\prime \prime \prime}+(f+g) f^{\prime \prime}-\left(f^{\prime}\right)^{2}-M^{2} f^{\prime}= \\
& \left(F^{\prime \prime}+G^{\prime \prime}\right) / 2+H\left(\left(F^{\prime}+G^{\prime}\right) / 2\right)-((F+ \\
& G) / 2)^{\wedge} 2-M^{\wedge} 2((F+G) / 2), R E S_{-} g=g^{\prime \prime \prime}+ \\
& (f+g) g^{\prime \prime}-\left(g^{\prime}\right)^{\wedge} 2-M^{\wedge} 2 g^{\prime}= \\
& \left(F^{\prime \prime}-G^{\prime \prime}\right) / 2+H\left(\left(F^{\prime}-G^{\prime}\right) / 2\right)-((F- \\
& G) / 2)^{\wedge} 2-M^{\wedge} 2((F-G) / 2) .
\end{aligned}
$$

Figures. 7 and 8 show the logarithmic of absolute values of Legendre coefficients as computed for several scaling factor $L$. The optimum $L$ for $N=20, M=0.1, R=0$ and $B=0.1$ is about $L=6$. Note that the curves for small $\mathrm{L}$ are well below those of the solid circles for $L=6$. Because $\left|L_{j}\left(2 \tanh \left(\frac{\eta}{L}\right)-1\right)\right| \leq 1$ for all real $\eta$, it follows that the error in truncating a spectral series is bounded by the sum of the absolute values of all the neglected coefficients (Boyd 2001).

Figures. 9 and 10 show the sensitivity of the Legendre basis to the choice of the map parameter $L$. For a given truncation $N$, there is an optimum $L$ which increases with increasing truncation. So, we observe that the choice of the map parameter $\mathrm{L}$ is not very critical. 


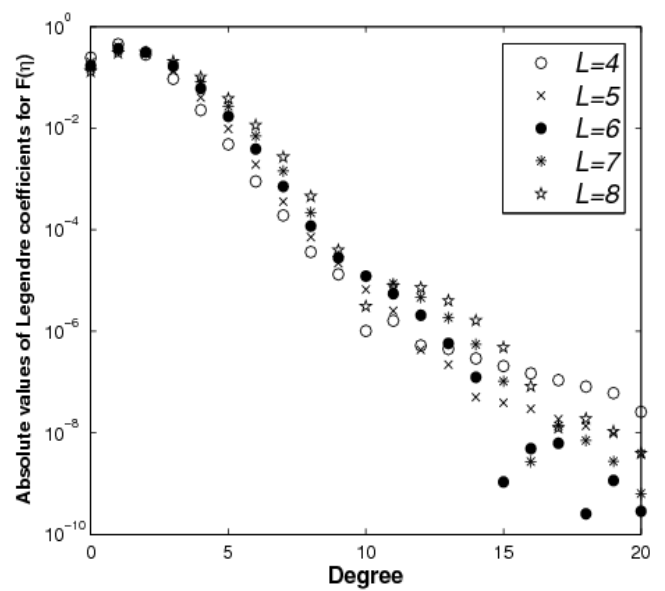

Fig. 7. The coefficients $\tilde{\mathbf{f}}_{\mathbf{j}}$ versus $\mathbf{j}$ for five different values of $L$. The solid circles show the best choice for $N=20$, which is $L=6$.

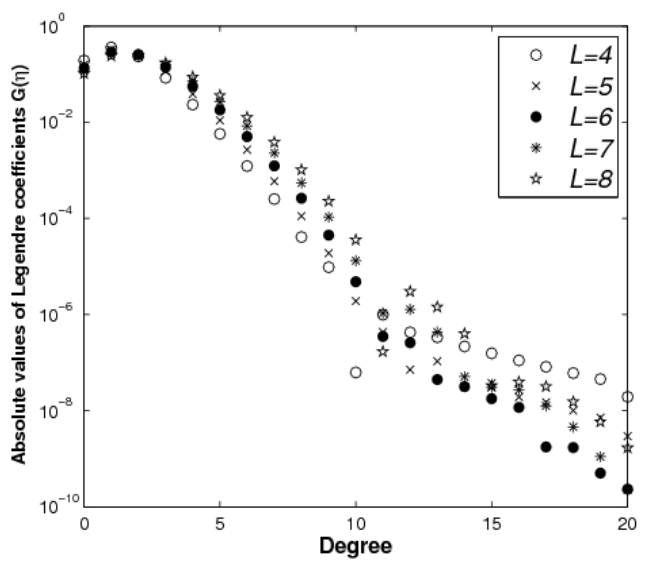

Fig.8. The coefficients $\widetilde{\boldsymbol{g}}_{\boldsymbol{j}}$ versus $\mathbf{j}$ for five different values of $\mathrm{L}$. The solid circles show the best choice for $N=20$, which is $L=6$.

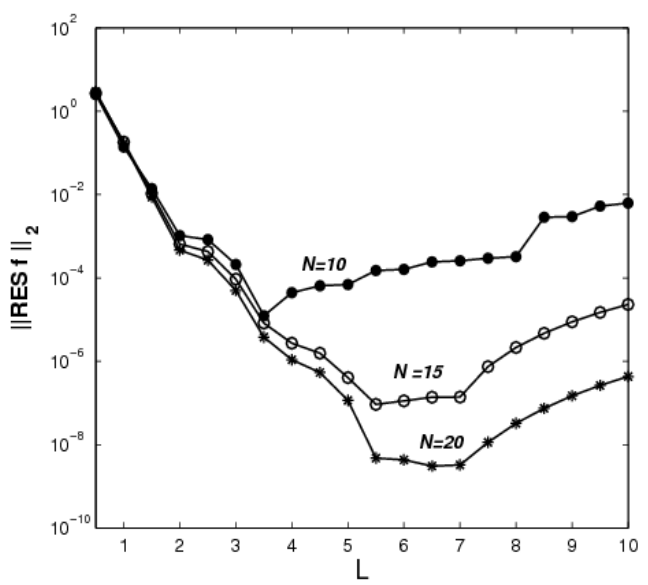

Fig. 9. Comparison of the residual errors for $f(\eta)$ with different choices of the parameter $L$ and the number of grid points $\mathrm{N}$ when $M=0.1, R=0$ and $B=0.1$.

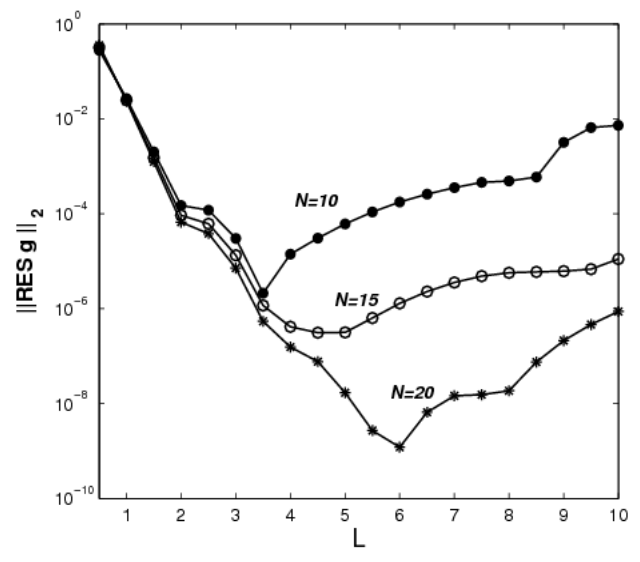

Fig. 10. Comparison of the residual errors for $g(\eta)$ with different choices of the parameter $L$ and the number of grid points $N$ when $M=0.1, R=0$ and $B=0.1$.

\section{CONCLUSIONS}

In this paper, a simple and accurate numerical algorithm based on Legendre pseudo-spectral method with a positive scaling factor and extrapolation for solving the system of nonlinear ordinary differential equations derived from similarity transform for the generalized threedimensional MHD flow over a porous stretching sheet is developed. This method reduces the solution of governing equations to the solution of a system of algebraic equations. The results are presented graphically and tabularly and the effect of the various pertinent parameters is discussed. An excellent agreement is seen between the results obtained by Wang, Ariel(2003), Ariel(2007), Hayat and presented method. The obtained results indicate that the method is a useful tool for the solution of nonlinear problems in fluid mechanics.

\section{ACKNOWLEDGEMENTS}

The authors are very much grateful to the referees for their valuable comments and suggestions for improving the paper.

\section{REFERENCES}

Ackroyd, J. (1978). On the steady flowproducedby a rotating discwith either surface suction or injection. J EngPhys 12, 207-220.

Adomian, G. (1994). Solving frontier problems of physics: the decomposition method. Dordrecht: Kluwer Academic Publishers.

Andersson, H. (1992). Mhd flow of a viscoelastic fluid past a stretching surface. ActaMech 95, 227-230.

Ariel, P. (2003). Generalized three-dimensional flow due to a stretching sheet. ZAMM 83, 844-852. 
Ariel, P. (2007). The three-dimensional flow past a stretching sheet and the homotopyperturba- tion method. Comput Math Appl 54, 920-925.

Ariel, P. D. (1994). Mhd flow of a viscoelastic fluid past a stretching sheet with suction. ActaMech 105, 49-56.

Boyd, J. (1982). The optimization of convergence for chebyshev polynomial methods in a unbounded domain. Commun Comput Phys 45, 43-79.

Boyd, J. (1987). Spectral methods using rational basis functions on an infinite interval. $J$ Com- put Phys 69, 112142.

Boyd, J. (1994). The rate of convergence of fourier coefficients for entire functions of infinite order with application to the weideman- clootsinh-mapping for pseudospectral com- putations on an infinite interval. J ComputPhys 110, 360-372.

Boyd, J. P. (2001). Chebyshev and Fourier Spectral Methods. Mineola NY: Dover Publications Inc.

Canuto, C., M. Y. Hussaini, A. Quarteroni, and T. A. Zang (1988). Spectral methods in fluid dynamics. New York: Springer-Verlag.

Chen, C. K. and M. Char (1988). Heat transfer of a continuous stretching surface with suction or blowing. J Math Anal Appl 135, 568-580.

Civalek, O. (2006). Harmonic differential quadrature-finite differences coupled approaches for geometrically nonlinear static and dynamic analysis of rectangular plates on elastic foundation. J Sound Vibr 294, 966-980.

Civalek, O. (2007). Nonlinear analysis of thin rectangular plates on winkler-pasternakelastic foundations by dsc-hdq methods. ApplMath Model 31, 606-624.

Clenshaw, C. and A. Curtis (1960). A method for numerical integration on an automatic computer. NumerMath 2, 197-205.

Crane, L. (1970). Flow past a stretching sheet. ZAMP 21, 645-647.

Datta, K. B. and B.M.Mohan (1995). OrthogonalFunctions in Systems and Control. USA: World Scientific River Edge NJ.

El-Mikkawy, M. and G. Cheon (2005). Combinatorial and hypergeometric identities via the legendrepolynomials-a computational ap- proach. Appl Math Comput 166, 181195
Gupta, P. and A. Gupta (1977). Heat and mass transfer on a stretching sheet with suction or blowing. Can J ChemEng 55, 744-746.

Hayat, T. and T. Javed (2007). On analytic solution for generalized three-dimensional mhdflow over a porous stretching sheet. PhysLettA 37, 243-250.

He, J. (1999). Variational iteration method: a kind of non-linear analytical technique: some ex- amples. Int J Nonlinear Mech 34, 699-708.

He, J. (2000). Variational iteration method for autonomous ordinary differential systems. ApplMath Comput 114, 115-123.

He, J. (2003). Homotopy perturbation method: A new nonlinear analytical technique. ApplMath Comput 135, 73-79.

He, J. (2004). The homotopy perturbation method for nonlinear oscillators with discontinuities. Appl Math Comput 151, 287-292.

Karmishin, A. V., A. I. Zhukov, and V. G. Kolosov (1990). Methods of dynamics calcu- lation and testing for thin-walled structures. Moscow: Mashinostroyenie.

Kelley, C. (1995). Iterative methods for linear and nonlinear equations. Philadelphia: PA: SIAM.

Liao, S. (2003). Beyond Perturbation: Introduction to the Homotopy Analysis Method. Boca Raton FL USA: Hall/CRCPress.

Mahanti, N.C. and P. Gaur (2009).Effects of Varying Viscosity and Thermal Conductivity on SteadyFree Convective Flow and Heat Transfer along an Isothermal Vertical Plate in the Presence of Heat Sink. J. Appl. Fluid.Mech 2, 23-28.

Maleknejad, K. and M. T. Kajani (2003). Solving second kind integral equation by galerkin methods with hybrid legendre and blockpulsefunctions. Appl Math Comput $145,623-629$.

Ortega, J. andW. Rheinboldt (1970). Iterative solution of nonlinear equations in several variables. New York: Academic Press.

Shen, J. and L. Wang (2009). Some recent advances on spectral methods for unbounded domains. CommunComputPhys 5, 195-241.

Snyder, M. (1966). Chebyshev methods in numerical approximation. Englewood Cliffs: N.J Prentice-Hall Inc. 
M. Heydari et al. / JAFM, Vol. 7, No. 3, pp. 473-483, 2014.

Troy, W., E. O. II, G. Ermountrout, and J. Keener (1987). Uniqueness of flow of a second order fluid past a stretching sheet. Quart ApplMath 44, 753-755.

Wang, C. (1984). The three-dimensional flow due to a stretching flat surface. Phys Fluids 27, 1915-1917.
Wang, C. (2002). Flow due to a stretching boundary with partial slip - an exact solution of the navier-stokes equations. ChemEngSci 57, 3745-3747.

Xu, H., S. J. Liao, and I. Pop (2006). Series solutions of unsteady boundary layer flow of a micropolar fluid near the forward stagnation point of a plane surface. ActaMech 184, 87-101. 